Article

\title{
Investigation of Optimal Coupling Velocities of the Sample and Sheath Flows for Hydrodynamic Focusing
}

\author{
Dingpeng Huang ${ }^{1}$, Hangzhou Wang ${ }^{1, *} \mathbb{C}$, Xiaoping Wang ${ }^{1}$, Kan Guo ${ }^{1}$, Zhuoli Yuan ${ }^{2}$, \\ Jiawang Chen ${ }^{1}$ (D) and Ying Chen ${ }^{1}$ (iD) \\ 1 Ocean College, Zhejiang University, Zhoushan 316021, China; huangdingpeng@zju.edu.cn (D.H.);

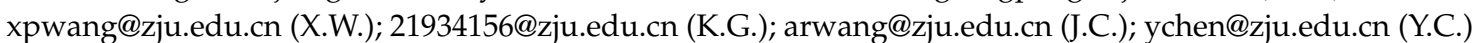 \\ 2 Polar Research Institute of China, Shanghai 200136, China; yuanzhuoli@pric.org.cn \\ * Correspondence: hangzhouwang@zju.edu.cn
}

Received: 6 July 2020; Accepted: 5 August 2020; Published: 12 August 2020

check for updates

\begin{abstract}
Focusing performance is a major concern for systems based on hydrodynamic focusing. In this study, the hydrodynamic focusing subsystem of a microscopic imaging system was analysed and modelled. The theoretical model was used to analyse the velocity and distribution range of sample particles in the focused sample flow in the micro-channel of the hydrodynamic focusing subsystem, when the velocities of the sample and sheath flows were varied. The results were used to optimise the coupling velocities of the sample and sheath flows for the microscopic imaging system, to keep working efficiency and image quality of the system simultaneously. An independent experiment was then conducted for verification, and the results agreed well with the theoretical investigation. The results of this study provide a general framework for adjusting the sample and sheath flow velocities to optimise the hydrodynamic focusing performance.
\end{abstract}

Keywords: hydrodynamic focusing; optimal coupling velocity; sample flow; sheath flow; microchannel

\section{Introduction}

Microfluidic focusing is used to arrange particles randomly distributed in fluid samples (e.g., water, blood) and it has been widely applied in many fields, such as biology, clinical research, and oceanology [1]. In flow cytometry, microfluidic focusing is used for counting, analysing, and sorting biological particles. Microfluidic focusing methods include electro-kinetic [2,3], acoustic [4], and hydrodynamic focusing [5]. Hydrodynamic focusing is based on fluid dynamics and offers two advantages over the other two methods: it does not need external forces (e.g., electro-kinetic, acoustic) or special requirements for the sample particles or fluids, which simplifies the system complexity [6], and it can operate effectively at a high flow rate to achieve a high throughput. These advantages indicate that hydrodynamic focusing can be used to develop highly efficient and inexpensive micro-flow cytometers [7].

In a hydrodynamic focusing system, the sample flow is generally enclosed and focused by the outer sheath flow to form a sample flow core, which passes through a predetermined area for further investigation. The characteristics (e.g., width, velocity) of the sample flow core, which significantly determine the system performance, mainly depend on the hydrodynamic characteristic of the flows in the system, microstructure of the flow channel, etc. Some studies have focused on investigating the theoretical hydrodynamic mechanisms of fluids to optimise the sample flow core for different applications [8,9]. Wang et al. analysed the focusing force exerted on the sample flow and particles in the sample flow core and found that it included the flow-induced drag force and inertial lift force. They also developed an inertial microfluidic focusing chip that only uses the inertial lift force to focus sample particles into a single position [10]. Panwar et al. examined the focusing performance of their 
microfluidic focusing chip at different Reynolds numbers (Re) and observed that the performance increased with Re [11]. Many other studies have considered the design or optimisation of flow channel microstructures $[12,13]$. Lee et al. designed a microfluidic focusing flow channel after numerically modelling the focusing effect of different structures and the relative flow rate between the sheath and sample flows [14]. They also examined the focusing performance of different types of micro-channels (including symmetric and asymmetric) with different aspect ratios, then proposed an analytical method for predicting the location and width of the sample flow core in different micro-channels [15]. John et al. presented a micro-channel with both curved and straight sections to realise microfluidic inertial focusing. They demonstrated that this channel could passively focus sample particles based on inertia without the assistance of a sheath fluid [16]. Zhao et al. modelled the focusing behaviour of a chip-based microfluidic hydrodynamic structure. They developed a novel structure to achieve an effective focusing performance at a high sample flow rate and reported that the difference in velocities of the sheath and sample flows should be minimised where they meet to prevent potential mixing [17].

A microscopic imaging system is currently in development that can autonomously collect images of phytoplankton $10-150 \mu \mathrm{m}$ in size from water samples. This system has adopted hydrodynamic focusing to arrange disordered particles in the water sample and pass them through the predetermined imaging area in sequence at an appropriate velocity. The velocities of the sample and sheath flows are adjusted at the micro-channel inlet. However, previous studies have mostly considered focusing the sample flow into a single-particle stream or simply noted the range of flow rates employed by a hydrodynamic focusing system. To the best of our knowledge, no studies have tried to improve the system efficiency by optimising the coupling velocities of the sample and sheath flows. In this study, we analysed and modelled the hydrodynamic focusing subsystem of the microscopic imaging system. The changes in velocity and distribution of sample particles in the imaging area with the velocities of the sample and sheath flows were theoretically simulated to obtain the optimal coupling velocities of these flows. The theoretical results were compared with those of a laboratory experiment for validation.

\section{System Overview}

As shown in Figure 1, the microscopic imaging system is primarily composed of a hydrodynamic focusing subsystem and optical imaging subsystem. The former is used to arrange disordered particles in the water sample and pass them through the imaging area in sequence. The latter takes images of the particles as they pass through the imaging area.

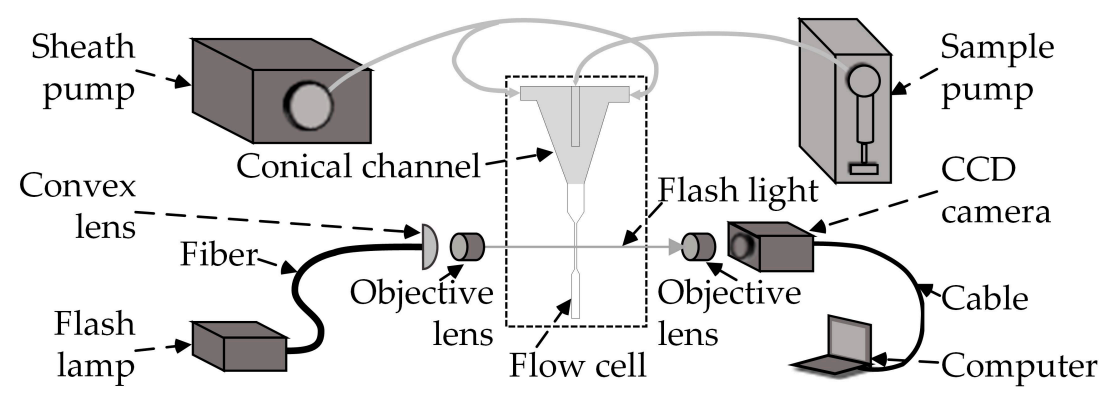

Figure 1. Schematic diagram of the configuration of the microscopic imaging system.

The hydrodynamic focusing subsystem comprises a sample pump, sheath flow pump, flow cell, and conical channel. A water sample is taken by the sample pump and then discharged very slowly via a fine needle at the central axis of the conical channel. A programmable syringe pump (ZSB-SY03, RUNZE Fluid Co., Ltd., Nanjing, Jiangsu, China) equipped with a $500 \mu \mathrm{L}$ syringe was selected as the sample pump. The number of steps and speed of each step of the pump can be adjusted separately via a serial interface (i.e., RS-232 interface), which determines the flow rate of the pump from $0.142 \mu \mathrm{L} / \mathrm{s}$ to $36.1 \mu \mathrm{L} / \mathrm{s}$. At the outlet of the fine needle, the water sample is enclosed by a much faster outer 
sheath fluid generated by the sheath flow pump. The sheath flow pump was a peristaltic pump (BT100-2J, Longer Precision Pump Co., Ltd., Baoding, Hebei, China) with a flow rate of 0.3-366.6 $\mu \mathrm{L} / \mathrm{s}$ and precision of $0.3 \mu \mathrm{L} / \mathrm{s}$. The sheath flow aligns the particles of the injected water sample in sequence and keeps them in the centre. These particles enter the flow cell underneath the conical channel, and they are focused along the centre axis of the micro-channel, ideally. The silicon flow cell has a square micro-channel with a cross-section of $200 \times 200 \mu \mathrm{m}$, which is wide enough to allow particles as large as $150 \mu \mathrm{m}$ in diameter.

The optical imaging subsystem comprises a charge-coupled device (CCD) camera, a flash lamp, two objective lenses, and a convex lens. The CCD camera $(\mathrm{GC} 1380 \mathrm{H}$, Allied Vision Technologies Canada Inc., Burnaby, BC, Canada) is equipped with an objective lens having a magnification of 10x, and it is focused on the centre axis of the micro-channel (i.e., imaging area) to take images of the particles as they pass through. The camera has an image resolution of $1360 \times 1024$ pixels, and each pixel has a size of $6.45 \times 6.45 \mu \mathrm{m}$. These dimensions were appropriate for our study because the target particles had a size of 10-150 $\mu \mathrm{m}$. The light emitted by the flash lamp (L9455-01, Hamamatsu Photonics Co., Ltd., Shizuoka, Japan) is guided by an optical fibre and is focused on the imaging area by the lens group (i.e., the combination of the objective lens and convex lens) to illuminate the imaging area. The flash lamp has a maximum repetition rate of $182 \mathrm{~Hz}$ and maximum input energy of $27.5 \mathrm{~mJ}$ per flash, which are vital to reducing the potential smear of particles in the images.

Because of the working mechanism of the system described above, particles in the micro-channel are always in motion. To take a clear image of these moving particles, two conditions need to be met. First, the particle should be within the depth of field (DOF) of the imaging device (i.e., the CCD camera and objective lens), which refers to the distance interval between the nearest and farthest objects sharply focused in an image. Second, the velocity of a particle in the imaging area should be optimised. An overly slow velocity reduces the working efficiency of the system, whereas an excessively fast velocity smears the particles in the image and reduces the imaging quality. The particle velocity is primarily determined by the velocities of the sample and sheath flows, geometry of the flow channel, etc. To ensure the working efficiency and image quality of the system, these two requirements must be satisfied simultaneously. We did this by optimising the coupling velocities of the sample and sheath flows via numerical modelling and validating the simulation results through a laboratory experiment.

\section{Theoretical Optimisation of the Coupling Velocities}

A theoretical model was built to represent the hydrodynamic focusing subsystem. Different combinations of the sample and sheath flow velocities were inputted to the model to investigate changes in the focusing performance (i.e., velocity and distribution range) of particles in the micro-channel and to determine the optimal coupling velocities of the sample and sheath flows.

\subsection{Theoretical Model}

As shown in Figure 2, a symmetric 3D model was adopted because the flow cell in the hydrodynamic focusing subsystem is symmetric and the conical channel is axisymmetric. These two parts were treated as a whole because they are tightly and physically connected. The model had two inlets and one outlet. One inlet was located at the axis of the conical channel to represent the entrance of the sample flow. It was surrounded by another inlet, which represented the entrance of the sheath flow. The outlet was placed at one end of the flow cell. Because the sample and sheath flows were independent, their velocities could be adjusted separately to examine the focusing performance. The relevant constants used in this model are listed in Table 1. 


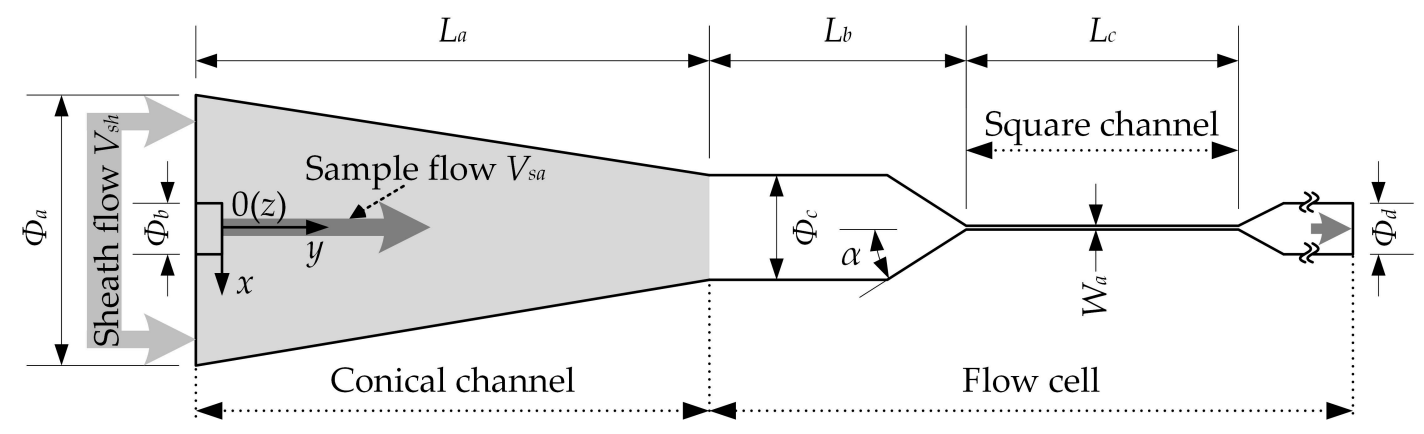

Figure 2. Cross sectional view of the theoretical model, indicating its geometric dimensions, inlet and outlet of the flows. $\Phi$ indicates diameter, $L$ indicates length, and $W$ indicates width.

Table 1. Constants adopted in the theoretical model.

\begin{tabular}{cccc}
\hline Description & Expression & Description & Expression \\
\hline$L_{a}$ & $9.9 \mathrm{~mm}$ & $\Phi_{a}$ & $5.85 \mathrm{~mm}$ \\
$L_{b}$ & $5 \mathrm{~mm}$ & $\Phi_{b}$ & $1 \mathrm{~mm}$ \\
$L_{c}$ & $5.75 \mathrm{~mm}$ & $\Phi_{c}$ & $2 \mathrm{~mm}$ \\
$\alpha$ & $30^{\circ}$ & $\Phi_{d}$ & $1 \mathrm{~mm}$ \\
$W_{a}$ & $0.2 \mathrm{~mm}$ & & \\
\hline
\end{tabular}

In the micro-channel, the sample particle goes along the focused sample flow. To ensure a clear image of a moving particle, its motion in the micro-channel should be very stable and smooth; this requires laminar flow. Re was adopted as an indicator of laminar flow. It measures the ratio of inertial to viscous forces acting on particles flowing in a channel:

$$
\operatorname{Re}=\frac{\rho U_{\mathrm{m}} D_{h}}{\mu},
$$

where $\rho$ is the fluid density, $\mu$ is the coefficient of viscosity of the fluid, $U_{m}$ is the maximum velocity of the fluid, and $D_{h}$ is the hydraulic diameter of the channel. This can be expressed as

$$
D_{h}=\frac{4 A}{P_{w}}=W_{a}
$$

where $A$ is the cross-sectional area of the channel, $P_{w}$ is the wetted perimeter, and $W_{a}$ is the channel width. By replacing $D_{h}$ with Equation (2) and $U_{m}$ with the maximum velocity $V_{\max }$ in the micro-channel, Equation (1) can be rewritten as

$$
\operatorname{Re}=\frac{\rho V_{\max } W_{a}}{\mu} .
$$

Two parameters were used to evaluate the focusing performance: the width of the focused sample flow $W_{s a}$ and particle velocity $V_{p}$. Because the micro-channel had a laminar flow, the motion of the sample flow could be described via Navier-Stokes (N-S) equations. Three assumptions were used to investigate $W_{s a}[15]$ :

1. The fluid is Newtonian. For a Newtonian fluid subjected to shear, the resulting strain rate $\varepsilon_{x y}$ is linearly related to the applied stress $\tau_{x y}$, which is numerically expressed as $\tau_{x y}=2 \mu \varepsilon_{x y}$ [18].

2. The flow in the micro-channel is steady.

3. The density of the fluid in the channel is uniform.

Based on the above assumptions and theory of hydrodynamics, $W_{s a}$ primarily depends on the width of the square micro-channel $W_{a}$ and velocities of the sample flow $V_{s a}$ and sheath flow $V_{s h}$. 
According to the hydrodynamics principle, in a straight square channel (i.e., square channel in Figure 2), the velocity profile $V$ of a fully developed laminar flow can be formulated as [19]

$$
V=\frac{4 W_{a}^{2}}{\mu \pi^{3}}\left(-\frac{d P}{d y}\right) \sum_{n=0}^{\infty} \frac{(-1)^{n}}{(2 n+1)^{3}}\left\{1-\frac{\cosh \left[(2 n+1) \pi x / W_{a}\right]}{\cosh [(2 n+1) \pi / 2]}\right\} \cos \frac{(2 n+1) \pi z}{W_{a}},
$$

where $x, y, z$ are the coordinates (Figure 2), $W_{a}$ is the channel width, and $P$ is the pressure. In a laminar flow, the velocity is along the axial direction of the channel. In each cross-section of the square channel, the velocity gradually decreases from the maximum at the centre to zero at the inner surface of channel.

\subsection{Theoretical Simulation}

\subsubsection{Parameters for Guiding and Evaluating the Theoretical Simulation}

Two parameters were adopted to evaluate the results of the theoretical simulation: the DOF of the imaging device and optimal particle velocity $V_{p \_o p}$. For the optical imaging subsystem, the DOF can be calculated as

$$
\mathrm{DOF}=\frac{\lambda \cdot n}{N A^{2}}+\frac{p \cdot n}{M \cdot N A},
$$

where $\lambda$ is the wavelength of light with a value of $550 \mathrm{~nm}, n$ is the refractive index of water and glass with a value of 2, and $p$ is the pixel size of the CCD camera (i.e., $6.45 \mu \mathrm{m}$ ). NA and $M$ are the numerical aperture and magnification, respectively, of the objective lens with values of 0.25 and 10 , respectively. Thus, the DOF was calculated to be $22.76 \mu \mathrm{m}$.

$V_{p \_o p}$ represents the critical particle velocity; above this value, the smear of the particle in the image collected by the CCD camera will be serious and unacceptable. Because there is no rigorous definition for calculating $V_{p_{-} \_p}$, an empirical formula was adopted to approximate it. From Olson et al. [20], a smear of 7.5 pixels was thought to be acceptable for sample particle (i.e., beads) as small as $20 \mu \mathrm{m}$. In this study, since the size of the smallest sample particle (i.e., $10 \mu \mathrm{m}$ ) was half of $20 \mu \mathrm{m}$, a smear of 3.75 pixels was thought to be appropriate. Therefore,

$$
3.75 e=M \cdot t \cdot V_{p \_o p}
$$

Conversely,

$$
V_{p_{-} o p}=\frac{3.75 e}{M \cdot t},
$$

where $e$ and $t$ are the pixel size and exposure time of the camera, respectively. For the optical imaging subsystem, a flash lamp was used instead of the shutter of the CCD camera to control the exposure time because the emission pulse duration of the former (i.e., $~ 1 \mu \mathrm{s}$ ) was far shorter than the shutter duration of the camera (i.e., $10 \mu \mathrm{s}$ ). By substituting the values of $1 \mu \mathrm{s}$ and $6.45 \mu \mathrm{m}$ into $t$ and $e$, respectively, in Equation (7), $V_{p_{-} o p}$ was calculated to be $2 \mathrm{~m} / \mathrm{s}$.

\subsubsection{Details of the Theoretical Simulation}

We investigated the motion of particles in the micro-channel following the theoretical model in Section 3.1 by using COMSOL Multiphysics (COMSOL Inc.). The sheath and sample flows were modelled in the laminar flow regime and were considered incompressible. The sample flow velocity was modelled at five values from $1.8 \mathrm{~mm} / \mathrm{s}$ to $9 \mathrm{~mm} / \mathrm{s}$, which corresponded to flow rates from 1.42 to $7.10 \mu \mathrm{L} / \mathrm{s}$ of the sample pump. At each sample flow velocity, the sheath flow velocity was modelled at five values of $1,1.5,2,3$, and $4 \mathrm{~mm} / \mathrm{s}$, which corresponding to flow rates of 25.5, 38.2, 51.0, 76.5, and $102 \mu \mathrm{L} / \mathrm{s}$ for the sheath flow pump. The velocities of the sample and sheath flows were selected to ensure that $V_{p_{-} o p}$ was within the velocity range of particles in the micro-channel. Each sample particle with the same velocity was distributed at a grid point of the inlet. All particles were released simultaneously (i.e., $t=0 \mathrm{~s}$ ) at the beginning of the simulation to trace their range. Because the sample 
flow and sheath flow velocities each had five test values, 25 groups of simulations were conducted in total. For each simulation, the particle distribution range and velocity and the flow velocity profile were recorded to evaluate the focusing performance of the hydrodynamic focusing subsystem. The relevant constants used in this simulation are listed in Table 2.

Table 2. Constants of the liquid and sample particles used in the theoretical simulation.

\begin{tabular}{cc}
\hline Description & Expression \\
\hline Density (liquid) & $1 \times 10^{3} \mathrm{~kg} / \mathrm{m}^{3}$ \\
Viscosity (liquid) & $1 \times 10^{-3} \mathrm{~Pa} \mathrm{~s}$ \\
Density (particle) & $1050 \mathrm{~kg} / \mathrm{m}^{3}$ \\
Diameter (particle) & $1 \times 10^{-5} \mathrm{~m}$ \\
Temperature & $293.15 \mathrm{~K}$ \\
\hline
\end{tabular}

\subsection{Theoretical Simulation Results}

\subsubsection{Sample Particle Velocity $V_{p}$}

In this study, the sample particle velocity $V_{p}$ in the micro-channel primarily depended on the sample flow velocity $V_{s a}$ and sheath flow velocity $V_{s h}$. To determine $V_{p_{-} o p}$, we needed to evaluate how $V_{p}$ is affected by $V_{s a}$ and $V_{s h}$. We observed that the flow velocity $V$ increased rapidly from the conical channel to the square micro-channel and remained almost steady for most of the micro-channel (Figure 3a). The flow velocity $V$ in the micro-channel gradually decreased from the maximum value at the centreline (i.e., $x=0, z=0$ ) to zero at the inner surface of the channel in a parabolic manner (Figures $3 \mathrm{~b}$ and 4 ), at each horizontal cross section the micro-channel. Therefore, a polynomial function could be adopted to represent $V$ as a function of $x$ by:

$$
V(x)=a x^{2}+b x+c,
$$

where $a, b$, and $c$ are the least-squares fitted coefficients (Table 3). And the goodness of the fit indicated by $R^{2}$ are all better than 0.9715 . These results agree with theoretical analysis in Section 3.1 and indicate that the micro-channel has a fully developed laminar flow [19]. The sample particles were closely focused around the central axis of the micro-channel (Figure 3c,d), which indicates that the hydrodynamic focusing subsystem functioned well. Meanwhile, the particle velocity was very close to the flow velocity in the micro-channel.

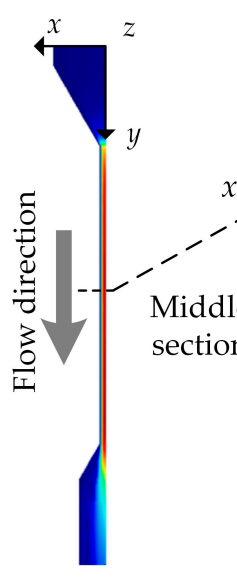

(a)

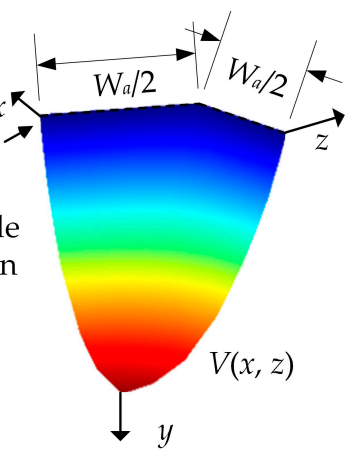

(b)

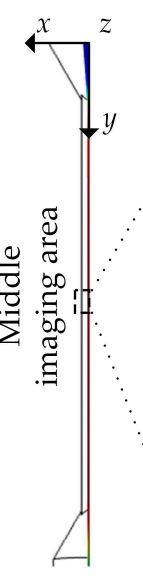

(c)

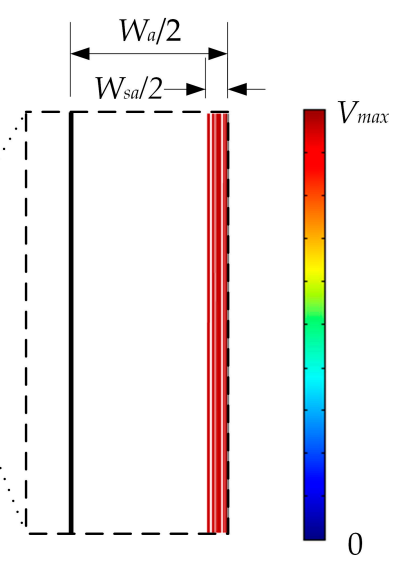

(d)

Figure 3. Flow velocity profile at (a) vertical cross section and (b) horizontal cross section of the micro-channel. Sample particle velocity profile at (c) side view and (d) the middle imaging area of the micro-channel. 


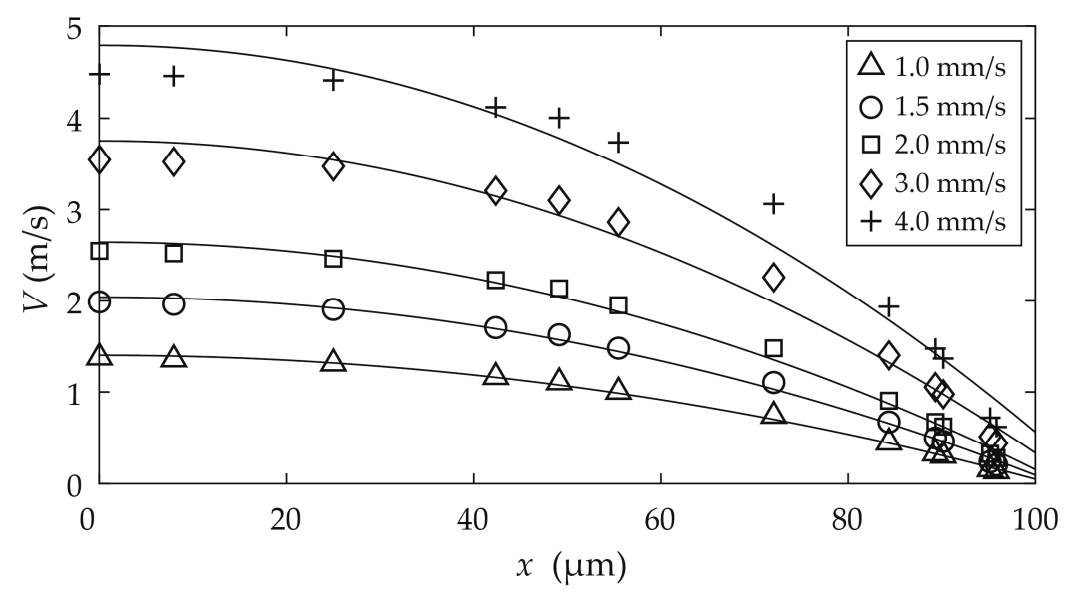

Figure 4. Flow velocity $V$ as a function of $x$ for five sheath flow velocities $V_{s h}$ at the sample flow velocity $V_{s a}$ of $1.8 \mathrm{~mm} / \mathrm{s}$.

Table 3. The fitted coefficients of flow velocity $V$ versus $x$ at five demonstrative sheath flow velocities $V_{s h}$ at the sample flow velocity $V_{s a}$ of $1.8 \mathrm{~mm} / \mathrm{s}$.

\begin{tabular}{ccccc}
\hline $\boldsymbol{V}_{\text {sh }}(\mathrm{mm} / \mathbf{s})$ & $\boldsymbol{a}$ & $\boldsymbol{b}$ & $\boldsymbol{c}$ & $\boldsymbol{R}^{\mathbf{2}}$ \\
\hline 1.0 & $-1.358 \times 10^{8}$ & $2.720 \times 10^{4}$ & 0.04044 & 0.9977 \\
1.5 & $-1.956 \times 10^{8}$ & $3.920 \times 10^{4}$ & 0.07649 & 0.9977 \\
2.0 & $-2.503 \times 10^{8}$ & $5.018 \times 10^{4}$ & 0.1286 & 0.9925 \\
3.0 & $-3.448 \times 10^{8}$ & $6.923 \times 10^{4}$ & 0.2786 & 0.9826 \\
4.0 & $-4.273 \times 10^{8}$ & $8.591 \times 10^{4}$ & 0.4718 & 0.9715 \\
\hline
\end{tabular}

As noted above, the sample particle velocities across the cross-section of the micro-channel were very similar. To facilitate the analysis, we selected the particle velocity at the intersection of the central axis and middle cross-section of the micro-channel to represent $V_{p}$. Figure 5 a shows that $V_{p}$ increased linearly with $V_{s h}$ for all values of $V_{s a}$. The increasing rate was very close for all $V_{s a}$ values, but the intercept increased with $V_{s a}$. Therefore, a linear function was adopted to represent $V_{p}$ as a function of $V_{s h}$ at each $V_{s a}$ :

$$
V_{p}\left(V_{s h}\right)=a_{s h} V_{s h}+b_{s h}
$$

where $a_{s h}$ and $b_{s h}$ are the least-squares fitted slope and intercept, respectively. The goodness of each fit was better than 0.9999 (Table 4). $V_{p}$ also increased in a strongly linear manner with increasing $V_{s a}$ at each $V_{s h}$, as shown in Figure $5 \mathrm{~b}$. Thus, we fitted a linear relationship to $V_{p}$ and $V_{s a}$ :

$$
V_{p}\left(V_{s a}\right)=a_{s a} V_{s a}+b_{s a}
$$

where $a_{s a}$ and $b_{s a}$ are the least-squares fitted slope and intercept, respectively. We found that the fitted intercept $b_{s h}$ increased slowly with increasing $V_{s a}$ (Table 4), while the intercept of $b_{s a}$ increased significantly with increasing $V_{s h}$ (Table 5). The fitted slope $a_{s h}$ was almost 40 times greater than the fitted slope $a_{s a}$, which indicated that the sample particle velocity was much more dependent on the sheath flow velocity than the sample flow velocity. This result was attributed to the fact that the sheath flow had a much greater flow rate than the sample flow in the micro-channel. 

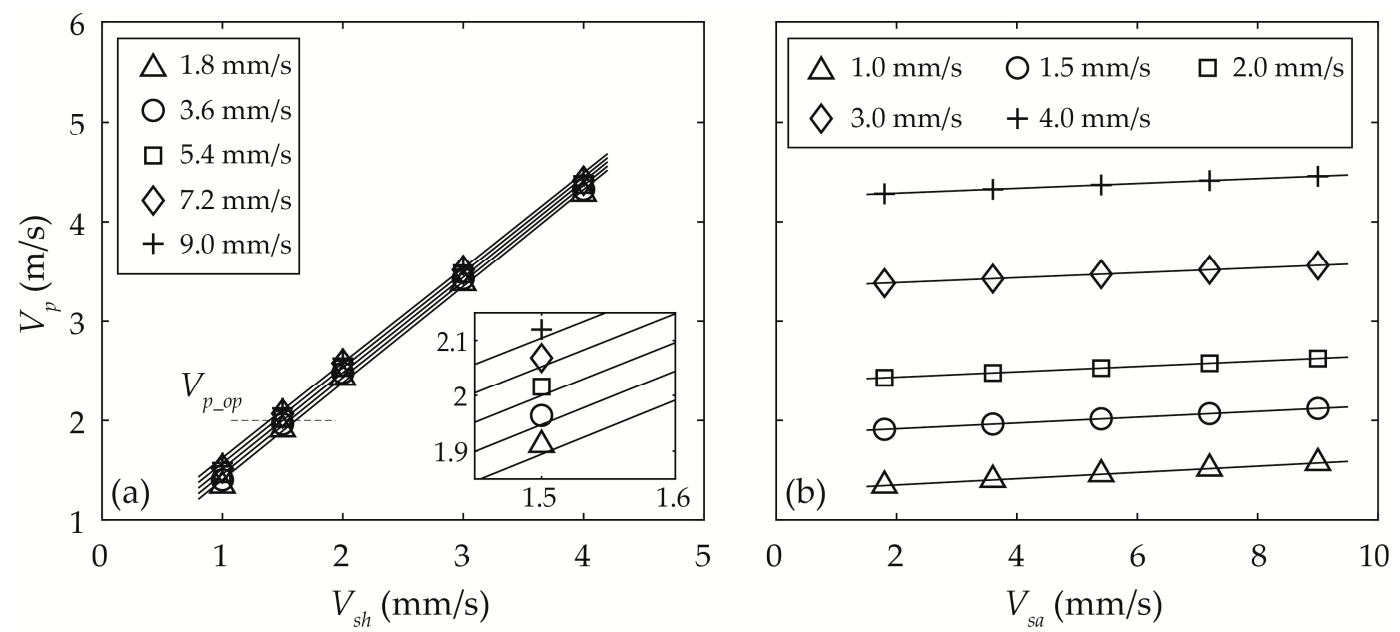

Figure 5. Sample particle velocity $V_{p}$ as a function of (a) sheath flow velocity $V_{s h}$ at five different sample flow velocities $V_{s a}$, and (b) $V_{s a}$ at five different $V_{s h}$. The dashed line in (a) represents the critical particle velocity $V_{p_{-} \text {op }}($ i.e., $2 \mathrm{~m} / \mathrm{s}$ ).

Table 4. The fitted coefficients of sample particle velocity $V_{p}$ versus sheath flow velocity $V_{s h}$ at five different sample flow velocities $V_{s a}$.

\begin{tabular}{cccc}
\hline $\boldsymbol{V}_{\boldsymbol{s} \boldsymbol{a}}(\mathbf{m m} / \mathbf{s})$ & $\boldsymbol{a}_{\boldsymbol{s h}}$ & $\boldsymbol{b}_{\boldsymbol{s h}}$ & $\boldsymbol{R}^{\mathbf{2}}$ \\
\hline 1.8 & 0.9710 & 0.4379 & 0.9999 \\
3.6 & 0.9664 & 0.4980 & 0.9999 \\
5.4 & 0.9624 & 0.5562 & 0.9999 \\
7.2 & 0.958 & 0.6155 & 0.9999 \\
9 & 0.9541 & 0.6731 & 0.9999 \\
\hline
\end{tabular}

Table 5. The fitted coefficients of sample particle velocity $V_{p}$ versus sample flow velocity $V_{s a}$ at five different sheath flow velocities $V_{s h}$.

\begin{tabular}{cccc}
\hline $\boldsymbol{V}_{\boldsymbol{s h}}(\mathbf{m m} / \mathbf{s})$ & $\boldsymbol{a}_{\boldsymbol{s} \boldsymbol{a}}$ & $\boldsymbol{b}_{\boldsymbol{s} \boldsymbol{a}}$ & $\boldsymbol{R}^{\mathbf{2}}$ \\
\hline 1.0 & 0.03126 & 1.295 & 0.9999 \\
1.5 & 0.02899 & 1.859 & 1 \\
2.0 & 0.02712 & 2.381 & 1 \\
3.0 & 0.02491 & 3.339 & 1 \\
4.0 & 0.02408 & 4.238 & 1 \\
\hline
\end{tabular}

\subsubsection{Width of the Sample Particle Stream $W_{s a}$}

Figure 3c,d show that the sample particles were focused around the central axis of the micro-channel for each combination of sample and sheath flow velocities. However, the width of the particle stream $W_{s a}$, which indicated the distribution range of the sample particles in the micro-channel (i.e., imaging area), changed differently depending on $V_{s h}$ and $V_{s a} . W_{s a}$ decreased with increasing $V_{s h}$, and the decreasing rate decreased with increasing $V_{s h}$ at each $V_{s a}$ (Figure 6a). In contrast, $W_{s a}$ increased with increasing $V_{s a}$, but the increasing rate decreased with increasing $V_{s a}$ for each $V_{s h}$ (Figure $6 \mathrm{~b}$ ). Hence, the following general power functions were used to represent $W_{s a}$ as functions of $V_{s h}$ for each $V_{s a}$ and $V_{s a}$ for each $V_{s h}$ :

$$
\begin{aligned}
& W_{s a}\left(V_{s h}\right)=f V_{s h}^{g}+h, \\
& W_{s a}\left(V_{s a}\right)=f V_{s a}^{g}+h,
\end{aligned}
$$

where $f, g$, and $h$ are the least-squares fitted coefficients. The goodness of the fit values are all better than 0.9981 (Tables 6 and 7). The power coefficient $g$ was relatively stable, and smaller and bigger than 
0 for Equations (11) and (12) separately. This phenomenon was in good agreement with the trend of $W_{s a}$ changing with $V_{s h}$ (Figure 6a) and $V_{s a}$ (Figure 6b), respectively. In contrast, the coefficient $f$ increased significantly with faster sample velocities $V_{s a}$ for Equation (11) and decreased significantly with faster sheath velocities $V_{s h}$ for Equation (12). These results indicated that a faster sheath flow and a slower sample flow were needed to narrow $W_{s a}$.
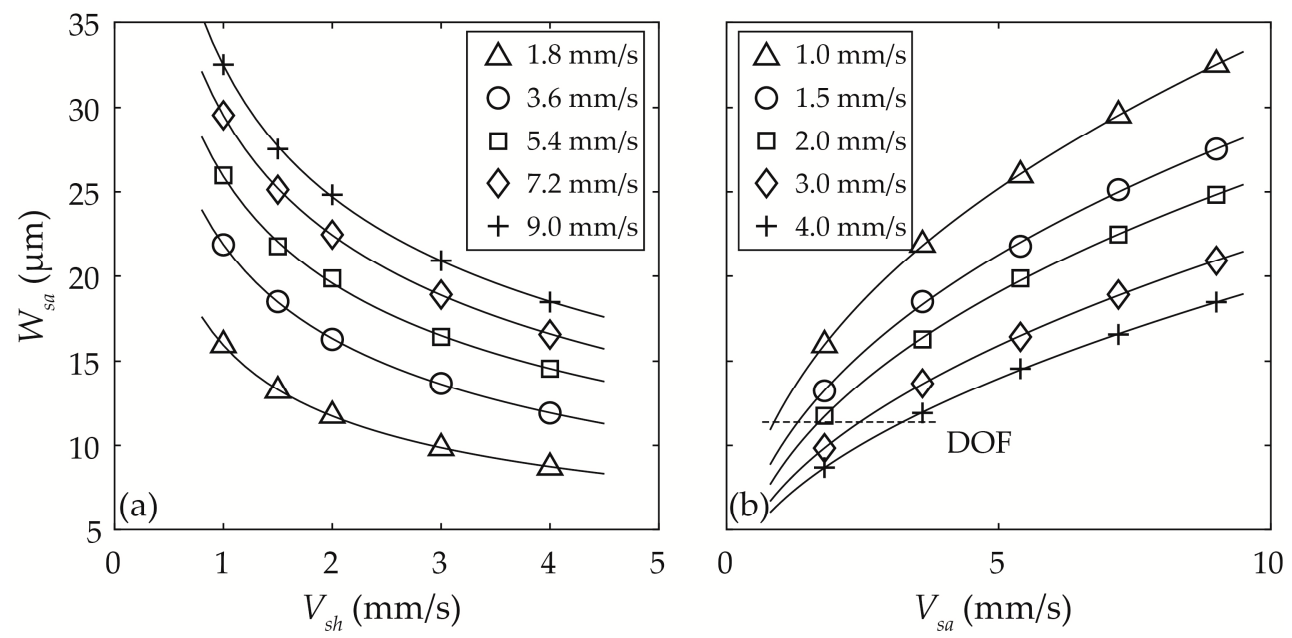

Figure 6. The width of sample particle stream $W_{s a}$ as a function of (a) sheath flow velocity $V_{s h}$ at five different sample flow velocities $V_{s a}$, and (b) $V_{s a}$ at five different $V_{s h}$. The dashed line in (b) is half of the depth of field (DOF) of the imaging device (i.e., $11.38 \mu \mathrm{m}$ ).

Table 6. The fitted coefficients of the width of sample particle stream $W_{s a}$ versus sheath flow velocity $V_{s h}$ at five different sample flow velocities $V_{s a}$.

\begin{tabular}{ccccc}
\hline$V_{\boldsymbol{s} a}(\mathrm{~mm} / \mathrm{s})$ & $f$ & $g$ & $\boldsymbol{h}$ & $\boldsymbol{R}^{\mathbf{2}}$ \\
\hline 1.8 & 14.74 & -0.4826 & 1.194 & 0.9984 \\
3.6 & 26.65 & -0.3364 & -4.780 & 0.9999 \\
5.4 & 30.82 & -0.3316 & -4.905 & 0.9981 \\
7.2 & 39.71 & -0.2841 & -10.18 & 0.9999 \\
9 & 37.95 & -0.3312 & -5.471 & 0.9996 \\
\hline
\end{tabular}

Table 7. The fitted coefficients of the width of sample particle stream $W_{s a}$ versus sample flow velocity $V_{s a}$ at five different sheath flow velocities $V_{s h}$.

\begin{tabular}{ccccc}
\hline$V_{\text {sh }}(\mathbf{m m} / \mathbf{s})$ & $f$ & $g$ & $h$ & $\boldsymbol{R}^{\mathbf{2}}$ \\
\hline 1.0 & 14.27 & 0.4037 & -2.128 & 0.9999 \\
1.5 & 12.77 & 0.3944 & -2.831 & 0.9992 \\
2.0 & 11.68 & 0.3953 & -3.006 & 0.9998 \\
3.0 & 7.725 & 0.4596 & -0.2743 & 0.9999 \\
4.0 & 6.006 & 0.4949 & 0.6476 & 0.9999 \\
\hline
\end{tabular}

\subsubsection{Optimisation of $V_{s h}$ and $V_{s a}$}

To ensure the working efficiency of the microscopic imaging system and take clear images of the sample particle, the sample particle velocity $V_{p}$ should be no more than $V_{p_{-} o p}$ (i.e., $2 \mathrm{~m} / \mathrm{s}$ ), and the distribution range of the sample particle $W_{s a}$ should be no more than the DOF (i.e., $22.76 \mu \mathrm{m}$ ) in the imaging area of the micro-channel. We investigated the optimal coupling velocities of the sheath and sample flows based on the above theoretical simulation to meet these two requirements concurrently.

We first set $V_{p}$ to $V_{p_{-} o p}$ in Equation (9) to obtain the corresponding $V_{s h}$ for each of the five simulated $V_{s a}$ values (i.e., five crosses on the dashed line with each fitted line in Figure 5a). The five pairs of $V_{s h}$ 
and $V_{s a}$ showed that $V_{s a}$ linearly decreased with faster $V_{s h}$ (triangles in Figure 7). Therefore, a linear fit was adopted to represent $V_{s a}$ as a function of $V_{s h}$ :

$$
V_{s a}=-36.11 V_{s h}+59.31
$$

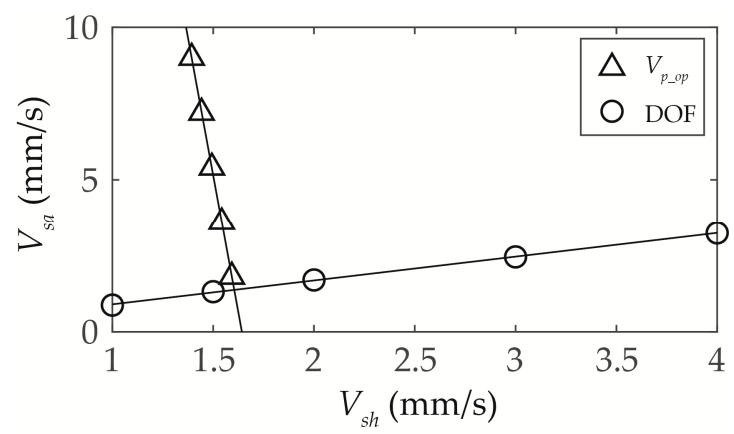

Figure 7. Sample flow velocity $V_{s a}$ as a function of sheath flow velocity $V_{s h}$ when velocity and distribution range of the sample particle are $V_{p_{-} o p}$ and DOF, respectively. The intersection of the two fitted lines is the optimised $V_{s h}$ and $V_{s a}$.

The goodness of the linear fit was better than 0.9999 as indicated by $R^{2}$. We then set $W_{s a}$ to the DOF in Equation (12) to obtain the corresponding $V_{s a}$ for each of the five simulated $V_{s h}$ values (i.e., five crosses on the dashed line with each fitted curve in Figure 6b). $V_{s a}$ linearly increased with faster $V_{s h}$ (circles in Figure 7). Thus, we applied a linear fit to the relationship between $V_{s a}$ and $V_{s h}$ :

$$
V_{s a}=0.7783 V_{s h}+0.1212 .
$$

The goodness of the linear fitting was better than 0.9995 as indicated by $R^{2}$. Then, we obtained the optimised $V_{s h}$ and $V_{s a}$, which were represented by the intersection of the two lines determined by Equation (13) and Equation (14) (Figure 7). The theoretical optimised $V_{s h}$ and $V_{s a}$ were 1.6045 and $1.3700 \mathrm{~mm} / \mathrm{s}$, respectively, which corresponded to sheath and sample flow rates of $40.9950 \mu \mathrm{L} / \mathrm{s}$ and $1.0755 \mu \mathrm{L} / \mathrm{s}$, respectively.

\section{Experimental Verification}

\subsection{Experiments}

Figure 8 shows an experimental prototype of the microscopic imaging system that was built to verify the optimal sample and sheath flow velocities from the above theoretical calculation. The key components were the same as those introduced in Section 2. The sample and sheath flow velocities at the entrance of the flow cell were adjusted with a syringe pump and peristaltic pump, respectively. The CCD camera was focused on the middle of the micro-channel (i.e., imaging area) and covered the entire channel width to monitor the movements of the flows and particles. Three types of water samples were fabricated and used in the experiment. Preliminary examinations showed that the smear of a polystyrene spherical bead (Thermo Fisher Scientific, Inc.) was much more significant than that of real phytoplankton; this was likely due to that the bead behaved like a micro-lens which focused the incident light at its focal point located on the axis passing by the center of the sphere. Thus, water sample 1 was made from polystyrene spherical beads dissolved in deionised water; this was used to mimic phytoplankton in water and examine how the sample particle velocity is affected by the sample and sheath flow velocities. The beads were spherical and had a nominal diameter of $12 \mu \mathrm{m}$ and density of $1055 \mathrm{~kg} / \mathrm{m}^{3}$. Water sample 2 was made of seawater containing Heterosigma akashiwo, which is a small phytoplankton with a diameter of $8-25 \mu \mathrm{m}$. This was used to test the image quality taken by the 
prototype with real phytoplankton. Water sample 3 was made with black dye ink to test the width of the sample flow core $W_{s a}$ in the micro-channel.

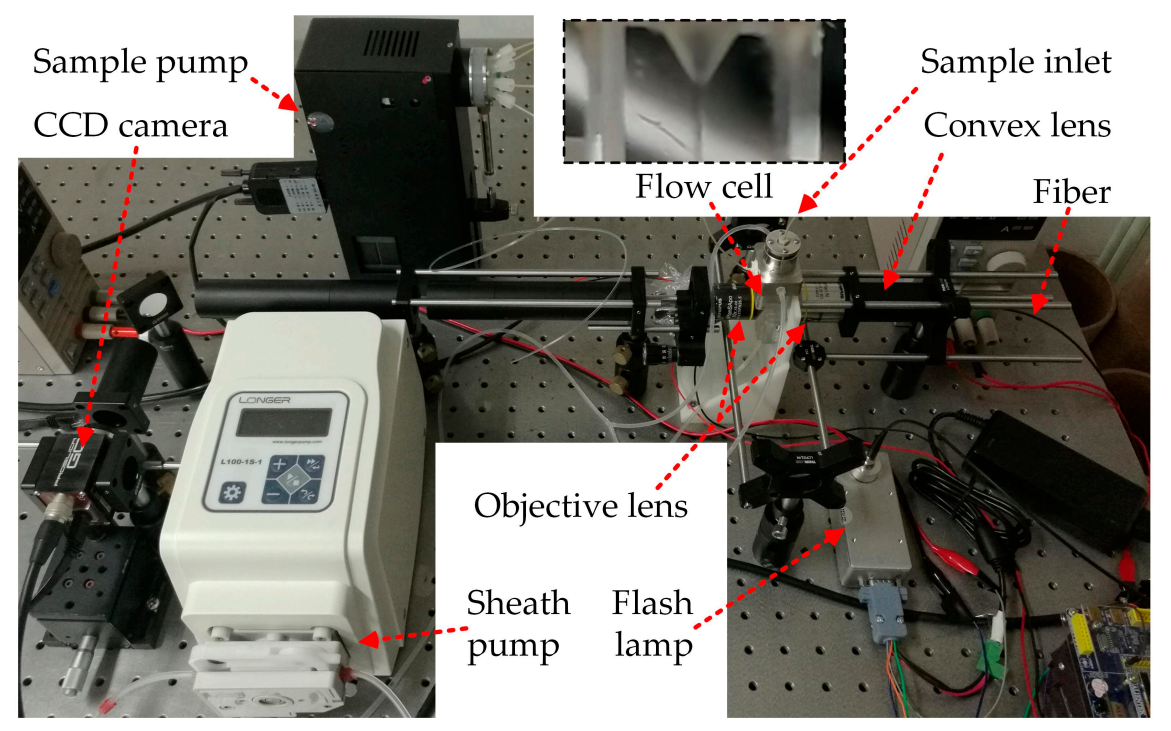

Figure 8. Experimental configuration of the microscopic imaging system. The flow cell is enlarged and shown in the dashed box.

The CCD camera was used to take images of water sample 1 in the micro-channel with varying sample and sheath flow rates to examine their effects on the sample particle velocity. The sample flow rate was set to $1.42,2.84,4.26,5.68$, and $7.10 \mu \mathrm{L} / \mathrm{s}$. For each sample flow rate, the sheath flow rate was set to $25.5,38.2,51.0,76.5$, and $102 \mu \mathrm{L} / \mathrm{s}$. Therefore, 25 groups of measurements were taken corresponding to these combinations. For each measurement, the CCD camera was set up to collect images at a frequency of $30 \mathrm{~Hz}$. The width of the sample flow core $W_{s a}$ was investigated in a very similar manner, except that sample 3 was used instead of sample 1 . We tested the image quality of the prototype with water sample 2 at a sample flow rate of $1.133 \mu \mathrm{L} / \mathrm{s}$ and sheath flow rate of $41.0 \mu \mathrm{L} / \mathrm{s}$, which were the closest to the theoretically optimal flow rates that could be generated by the prototype. Two different sheath flow rates of 25.5 and $76.5 \mu \mathrm{L} / \mathrm{s}$ were also considered for comparison.

\subsection{Experimental Results}

\subsubsection{Smear of Sample Particles}

Directly measuring the sample particle velocity in the micro-channel is demanding, and it requires expensive measurement devices. The above simulation had shown that the sample particle velocity in the imaging area was very steady (Figure 3). Alternatively, the smear of the sample particle can be considered proportional to the sample particle velocity; thus, it was used to evaluate the influence of the sample and sheath flow velocities. The measurements of water sample 1 showed that most of the polystyrene beads were imaged clearly (Figure 9). The smear length $L_{s}$ (i.e., length of the bright tail behind each bead) was quantified by the number of pixels along the horizontal axis passing through the centre of the particle. At each combination of velocities of the sample flow and sheath flow, we randomly selected 20 images and calculated $L_{s}$. The results denoted that $L_{S}$ was relatively stable and the absolute value of the discrepancy was no higher than $10 \%$ for each group of 20 images. The discrepancy was probably due to pulsation of the sheath pump and contrast change of the image. We observed that $L_{s}$ increased significantly with the sample and sheath flow velocities which agreed with the above assumption that $L_{S}$ was proportional to the sample particle velocity. 


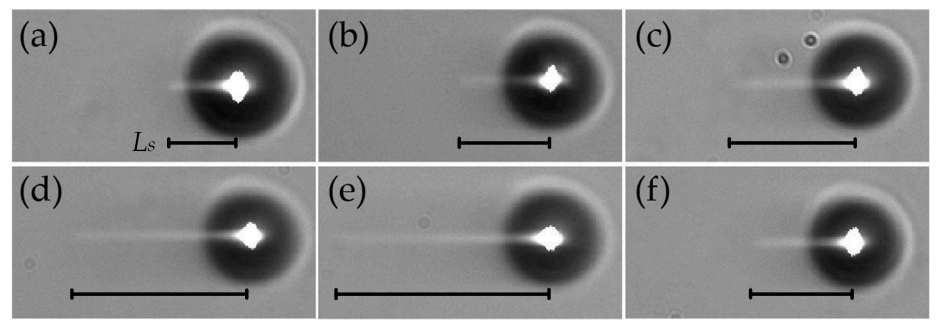

Figure 9. The smear length of water sample $1 L_{s}$ at five experimental sheath flow rates of (a) $25.5 \mu \mathrm{L} / \mathrm{s}$, (b) $38.2 \mu \mathrm{L} / \mathrm{s}$, (c) $51.0 \mu \mathrm{L} / \mathrm{s}$, (d) $76.5 \mu \mathrm{L} / \mathrm{s}$, (e) $102 \mu \mathrm{L} / \mathrm{s}$ and (f) the optimised flow rate of $41.0 \mu \mathrm{L} / \mathrm{s}$. The sample flow rate is $1.42 \mu \mathrm{L} / \mathrm{s}$.

To compare the experimental results (i.e., $L_{s}$ ) with the simulation results (i.e., $V_{p}$ ), we normalised each group of measurements as follows:

$$
X_{\text {nor }}=\left(X-X_{\text {avr }}\right) /\left(X_{\max }-X_{\min }\right),
$$

where $X_{n o r}$ is the normalised data, $X$ is the original data, $X_{\text {avr }}$ is the average of the group of measurements, and $X_{\max }$ and $X_{\min }$ are the maximum and minimum, respectively, of the group of measurements. After we normalised $L_{s}$, we found that the normalised smear length $L_{s}{ }^{\prime}$ increased linearly with increasing $V_{s h}$ and $V_{s a}$ (points with different marker styles in Figure 10). Therefore, $L_{s}{ }^{\prime}$ can be represented as the following linear functions of $V_{s h}$ and $V_{s a}$ :

$$
\begin{aligned}
& L_{s}^{\prime}\left(V_{s h}\right)=a_{s h}^{\prime} V_{s h}+b_{s h^{\prime}}^{\prime} \\
& L_{s}^{\prime}\left(V_{s a}\right)=a_{s a}^{\prime} V_{s a}+b_{s a}^{\prime}
\end{aligned}
$$

where $a_{s h}{ }^{\prime}, b_{s h}{ }^{\prime}, a_{s a}{ }^{\prime}$, and $b_{s a}{ }^{\prime}$ are the least-squares fitted coefficients (Tables 8 and 9). The goodness of the linear fitting indicated by $R^{2}$ for Equation (17) was not comparable to that of Equation (16), possibly ascribing to that the particle velocity in the micro-channel was dominated by the sheath flow. Similarly, we normalised $V_{p}$ according to Equation (15) and observed that the normalised sample particle velocity $V_{p}$ " also increased linearly with increasing $V_{s h}$ and $V_{s a}$. As a result, $V_{p}{ }^{\prime \prime}$ can be represented by the following linear functions of $V_{s h}$ and $V_{s a}$ (solid lines in Figure 10):

$$
\begin{aligned}
& V_{p}^{\prime \prime}\left(V_{s h}\right)=a_{s h}^{\prime \prime} V_{s h}+b_{s h^{\prime \prime}}^{\prime \prime} \\
& V_{p}^{\prime \prime}\left(V_{s a}\right)=a_{s a}^{\prime \prime} V_{s a}+b_{s a}^{\prime \prime}
\end{aligned}
$$

where $a_{s h}{ }^{\prime \prime}, b_{s h}{ }^{\prime \prime}, a_{s a}{ }^{\prime \prime}$, and $b_{s a}{ }^{\prime \prime}$ are the least-squares fitted coefficients (Tables 8 and 9). The goodness of the linear fitting are all better than 0.9984. Figure 10a shows that $L_{s}{ }^{\prime}$ (points with different marker styles) was uniformly distributed on both sides of each fitted $V_{p}$ " (solid line) for each $V_{s a}$. Meanwhile, the fitted slope $a_{s h}{ }^{\prime}$ was very close to the fitted slope of $a_{s h}{ }^{\prime \prime}$ (Table 8). Similarly, the general trend of $L_{s}{ }^{\prime}$ with $V_{s a}$ also agreed well with that of $V_{p}{ }^{\prime \prime}$ with $V_{s a}$ for each $V_{s h}$ (Figure 10b, Table 9). The root mean square error (RMSE) between $L_{s}{ }^{\prime}$ and $V_{p}{ }^{\prime \prime}$ was no higher than 0.02 for each group of $V_{s h}$ at each $V_{s a}$ (Figure 10a) or each group of $V_{s a}$ at each $V_{s h}$ (Figure 10b), which indicated that the laboratory experiment agreed well with theoretical simulation. 

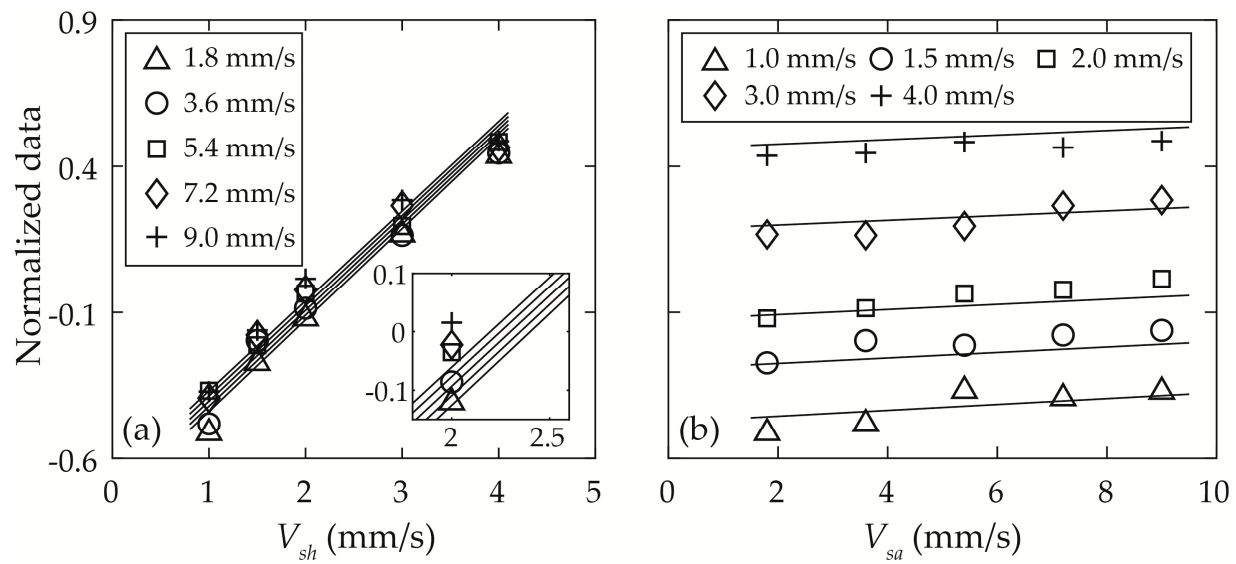

Figure 10. Normalized smear length $L_{s}{ }^{\prime}$ (the points with different marker styles) and normalized particle velocity $V_{p}$ " (the solid lines) as a function of (a) sheath flow velocity $V_{s h}$ at five different sample flow velocities $V_{s a}$, and (b) $V_{s a}$ at five different $V_{s h}$.

Table 8. The fitted coefficients of $L_{s}{ }^{\prime}$ and $V_{p}{ }^{\prime \prime}$ versus sheath flow velocity $V_{s h}$ at five different sample flow velocities $V_{s a}$.

\begin{tabular}{ccccccc}
\hline \multirow{2}{*}{$V_{\boldsymbol{s} \boldsymbol{a}}(\mathbf{m m} / \mathbf{s})$} & \multicolumn{3}{c}{$\boldsymbol{L}_{\boldsymbol{s}}{ }^{\prime}$ (Experiment) } & \multicolumn{3}{c}{$V_{\boldsymbol{p}}{ }^{\prime \prime}$ (Simulation) } \\
\cline { 2 - 7 } & $\boldsymbol{a}_{\boldsymbol{s} \boldsymbol{h}^{\prime}}$ & $\boldsymbol{b}_{\boldsymbol{s} \boldsymbol{h}^{\prime}}$ & $\boldsymbol{R}^{\mathbf{2}}$ & $\boldsymbol{a}_{\boldsymbol{s h}} \boldsymbol{\prime n}^{\prime}$ & $\boldsymbol{b}_{\boldsymbol{s h}}{ }^{\prime \prime}$ & $\boldsymbol{R}^{\mathbf{2}}$ \\
\hline 1.8 & 0.3069 & -0.7664 & 0.9895 & 0.3129 & -0.7514 & 0.9984 \\
3.6 & 0.2899 & -0.6976 & 0.9776 & 0.3114 & -0.7320 & 0.9985 \\
5.4 & 0.2789 & -0.6286 & 0.9961 & 0.3101 & -0.7133 & 0.9986 \\
7.2 & 0.2820 & -0.6211 & 0.9831 & 0.3087 & -0.6942 & 0.9987 \\
9 & 0.2816 & -0.5969 & 0.9813 & 0.3075 & -0.6756 & 0.9987 \\
\hline
\end{tabular}

Table 9. The fitted coefficients of $L_{s}{ }^{\prime}$ and $V_{p}{ }^{\prime \prime}$ versus sample flow velocity $V_{s a}$ at five different sheath flow velocities $V_{s h}$.

\begin{tabular}{ccccccc}
\hline \multirow{2}{*}{$\boldsymbol{V}_{\boldsymbol{s h}}(\mathbf{m m} / \mathbf{s})$} & \multicolumn{3}{c}{$\boldsymbol{L}_{\boldsymbol{s}}{ }^{\prime}$ (Experiment) } & \multicolumn{3}{c}{$\boldsymbol{V}_{\boldsymbol{p}}{ }^{\prime \prime}$ (Simulation) } \\
\cline { 2 - 7 } & $\boldsymbol{a}_{\boldsymbol{s} \boldsymbol{a}^{\prime}}$ & $\boldsymbol{b}_{\boldsymbol{s} \boldsymbol{a}^{\prime}}$ & $\boldsymbol{R}^{\mathbf{2}}$ & $\boldsymbol{a}_{\boldsymbol{s} \boldsymbol{} \boldsymbol{a}^{\prime \prime}}$ & $\boldsymbol{b}_{\boldsymbol{s} \boldsymbol{a}}{ }^{\prime \prime}$ & $\boldsymbol{R}^{\mathbf{2}}$ \\
\hline 1.0 & 0.02075 & -0.5371 & 0.7647 & 0.01008 & -0.4751 & 0.9999 \\
1.5 & 0.01336 & -0.2763 & 0.7921 & 0.009341 & -0.2933 & 1 \\
2.0 & 0.01864 & -0.1504 & 0.9760 & 0.008741 & -0.1253 & 1 \\
3.0 & 0.01864 & 0.1148 & 0.8940 & 0.008026 & 0.1834 & 1 \\
4.0 & 0.006681 & 0.4275 & 0.7177 & 0.007761 & 0.4731 & 1 \\
\hline
\end{tabular}

Figure 11 show the images of water sample 2 taken by the prototype at three different sheath flow rates. The smear of $H$. akashiwo was not as sensitive as that of the polystyrene bead to different shear flow velocities. At the theoretically optimal sample and sheath flow rates, the smear of the H. akashiwo was not very significant, and the shape of the phytoplankton could be clearly identified (Figure 11b). In contrast, with the faster sheath velocity, the shape of the phytoplankton became blurred (Figure 11c). The results indicated that the theoretically optimal sample and sheath flow rates obtained in this study are applicable to the microscopic imaging system. 


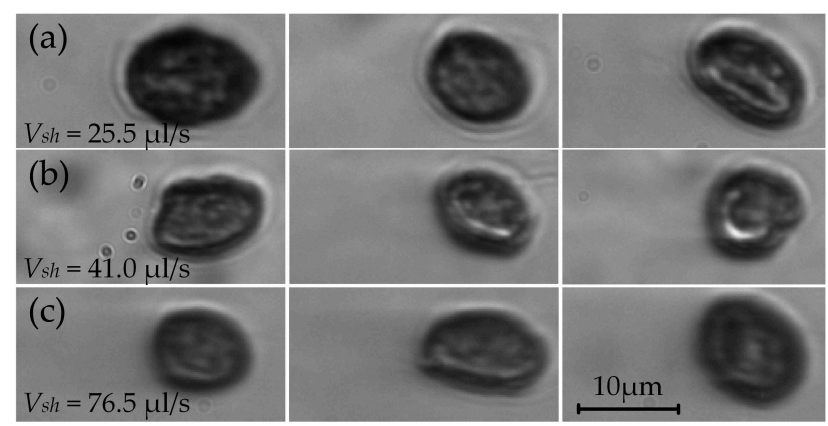

Figure 11. Images of water sample 2 at three different sheath flow rates of (a) $25.5 \mu \mathrm{L} / \mathrm{s}$, (b) $41.0 \mu \mathrm{L} / \mathrm{s}$ (i.e., the closest to the optimised sheath flow rate), and (c) $76.5 \mu \mathrm{L} / \mathrm{s}$. The sample flow rate is $1.133 \mu \mathrm{L} / \mathrm{s}$ (i.e., the closest to the optimised sample flow rate). The three photos in each row are taken under the same sample and sheath flow rates.

\subsubsection{Width of the Focused Sample Flow Stream $W_{s a}$}

Figure 12 shows that water sample 3 was precisely focused into a narrow stream in the micro-channel and passed through the imaging area very smoothly with each combination of the sample and sheath flow velocities. The focused stream was not at the central axis of the micro-channel because the fine needle used to discharge the sample flow was not installed exactly at the central axis of the conical channel. The width of the focused stream $W_{s a}$ changed with the sample flow velocity $V_{s a}$ and sheath flow velocity $V_{s h}$. $W_{s a}$ decreased gradually with increasing $V_{s h}$, and the decreasing rate decreased with increasing $V_{s h}$ for each $V_{s a}$ (Figure 13a). The experimental relationship between $W_{s a}$ and $V_{s a}$ agreed with that in the theoretical simulation: $W_{s a}$ increased with $V_{s a}$, but the increasing rate decreased with increasing $V_{s a}$ for each $V_{s h}$ (Figure 13b). Each group of experimental measurement data (discrete points in Figure 13) was uniformly distributed on both side of each fitted curve (solid curves in Figure 13) derived from the theoretical simulation in Section 3. The absolute value of the discrepancy between the theoretically calculated value and the experimental value was no higher than $6 \%$ for each group of sheath and sample flow velocities, which meant that our model could properly predict the width of the focused sample flow stream in the micro-channel.

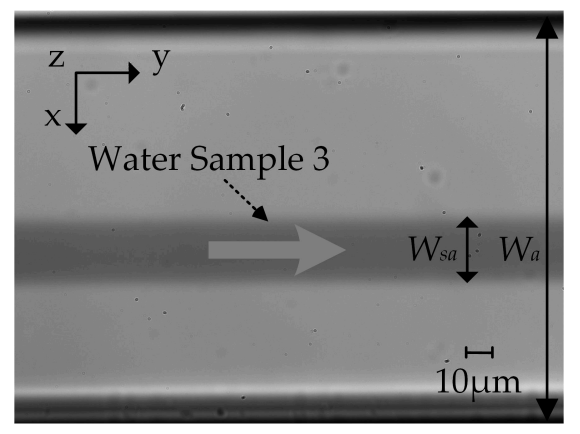

Figure 12. Images of sample 3 at the sheath flow rate of $25.5 \mu \mathrm{L} / \mathrm{s}$ and sample flow rate of $2.84 \mu \mathrm{L} / \mathrm{s}$. 

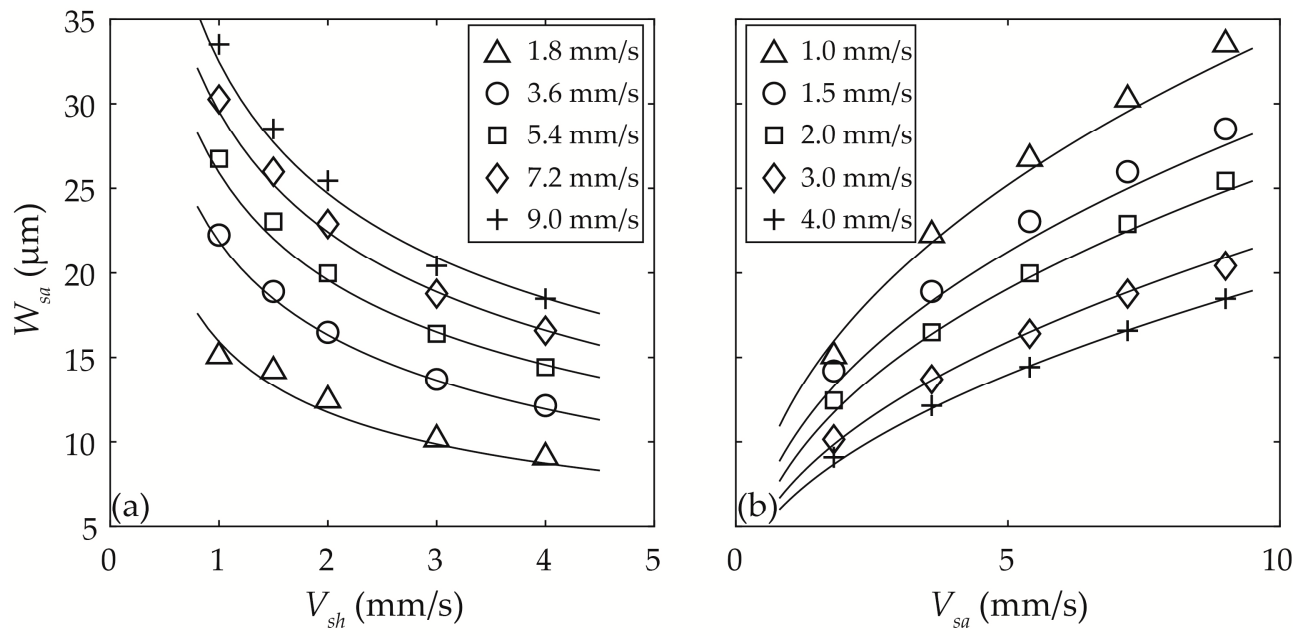

Figure 13. The width of sample particles stream $W_{s a}$ as a function of (a) sheath flow velocity $V_{s h}$ at five different sample flow velocities $V_{s a}$, and (b) $V_{s a}$ at five different $V_{s h}$. In this figure, the discrete points with different marker styles derive from the experimental measurement. Whereas, the solid curves derive from the theoretical simulation conducted in Section 3, which are the same as those shown in Figure 6.

\section{Conclusions}

The optimal coupling velocities of the sample and sheath flows of a microscopic imaging system were investigated to ensure its working efficiency and imaging quality. The hydrodynamic focusing subsystem was analysed and modelled with a symmetrical 3D model. The changes in the velocity $V_{p}$ and distribution range $W_{s a}$ of the sample particle in the imaging area with the sample flow velocity $V_{s a}$ and sheath flow velocity $V_{s h}$ were theoretically simulated. The results indicated that $V_{p}$ increased with both $V_{s a}$ and $V_{s h}$. The slope (i.e., increasing rate) of $V_{p}$ was much bigger with increasing $V_{s h}$ than with increasing $V_{s a}$, which indicates that $V_{p}$ is much more dependent on $V_{s h}$ than on $V_{s a} . W_{s a}$ decreased with increasing $V_{s h}$, and the decreasing rate decreased with increasing $V_{s h}$. However, $W_{s a}$ increased with $V_{s a}$, and the increasing rate decreased with increasing $V_{s a}$. These results were used to obtain the theoretically optimal coupling velocities of the sample and sheath flows. A prototype of the microscopic imaging system was built, and the changes in $V_{p}$ and $W_{s a}$ with different $V_{s a}$ and $V_{s h}$ were experimentally examined. The experimental results agreed well with the theoretical results. This indicates that the optimal coupling velocities of the sample and sheath flows are applicable to the microscopic imaging system.

Although the results obtained in this study are promising, several aspects might benefit from improvement. First, we investigated the optimal coupling velocities of the sample and sheath flows in the micro-channel using microparticle as small as $12 \mu \mathrm{m}$. Whereas, the size range of the sample particle for the microscopic imaging system was 10-150 $\mu \mathrm{m}$. The size change of the sample particle might lead to the variation of the particle Raynor number, which may lead to variation of the particle motion in the micro-channel and the optimal coupling velocities of the sample and sheath flows. Even though the influence of particle size may not be significant to this study [21], it should be investigated in more detail in future for rigor. Second, the direct measurement of velocities of the sample particle and flow in the micro-channel will be more intuitional to reflect the influence of velocities of the sample flow and sheath flow, and more convenient for the investigation of the optimal coupling velocities of the sample flow and sheath flow. Finally, fluorescent microbeads will be considered in future to examine the smearing effect of the beads under different velocities of the sample flow and sheath flow [22].

Author Contributions: Conceptualization, H.W. and X.W.; methodology, D.H., H.Z., and K.G.; software, D.H. and K.G.; investigation, D.H. and K.G.; resources, Z.Y. and J.C.; data curation, D.H. and H.W.; writing-original draft preparation, D.H. and H.W.; writing-review and editing, X.W., Z.Y., and J.C.; supervision, H.W. and X.W.; project administration, Y.C. All authors have read and agreed to the published version of the manuscript. 
Funding: This research was funded by Joint Zhoushan City and Zhejiang University Cooperation Project (Grant No. 2019C82089), National Natural Science Foundation of China (Grant No. 41976218), and the National Key Research and Development Program of China (Grant No. 2017YFC0307703).

Acknowledgments: The authors thank Mengmeng Tong (Ocean College, Zhejiang University) for providing phytoplankton during the experimental verification.

Conflicts of Interest: The authors declare no conflict of interest.

\section{References}

1. Altschuler, S.J.; Wu, L.F. Cellular heterogeneity: Do differences make a difference? Cell 2010, 141, 559-563. [CrossRef] [PubMed]

2. Das, D.; Biswas, K.; Das, S. A microfluidic device for continuous manipulation of biological cells using dielectrophoresis. Med. Eng. Phys. 2014, 36, 726-731. [CrossRef] [PubMed]

3. Jiang, H.; Weng, X.; Li, D. A novel microfluidic flow focusing method. Biomicrofluidics 2014, 8, 054120. [CrossRef]

4. Collins, D.J.; Morahan, B.; Garcia-Bustos, J. Two-dimensional single-cell patterning with one cell per well driven by surface acoustic waves. Nat. Commun. 2015, 6, 1-11. [CrossRef]

5. Ha, B.H.; Lee, K.S.; Jung, J.H. Three-dimensional hydrodynamic flow and particle focusing using four vortices Dean flow. Microfluid. Nanofluidics 2014, 17, 647-655. [CrossRef]

6. Versaci, M.; Palumbo, A. Magnetorheological fluids: Qualitative comparison between a mixture model in the extended irreversible thermodynamics framework and an Herschel-Bulkley experimental elastoviscoplastic model. Int. J. Non Linear Mech. 2020, 118, 103288. [CrossRef]

7. Luo, T.; Fan, L.; Zhu, R. Microfluidic single-cell manipulation and analysis: Methods and applications. Micromachines 2019, 10, 104. [CrossRef]

8. Hamilton, E.S.; Ganjalizadeh, V.; Wright, J.G. 3D Hydrodynamic Focusing in Microscale Optofluidic Channels Formed with a Single Sacrificial Layer. Micromachines 2020, 11, 349. [CrossRef]

9. Wang, Q.; Yuan, D.; Li, W. Analysis of hydrodynamic mechanism on particles focusing in micro-channel flows. Micromachines 2017, 8, 197. [CrossRef]

10. Wang, X.; Zandi, M.; Ho, C.C. Single stream inertial focusing in a straight microchannel. Lab A Chip 2015, 15, 1812-1821. [CrossRef]

11. Panwar, N.; Song, P.; Tjin, S.C. Sheath-assisted hydrodynamic particle focusing in higher Reynolds number flows. J. Micromechanics Microengineering 2018, 28, 105018. [CrossRef]

12. Chiu, Y.J.; Cho, S.H.; Mei, Z. Universally applicable three-dimensional hydrodynamic microfluidic flow focusing. Lab A Chip 2013, 13, 1803-1809. [CrossRef] [PubMed]

13. Mao, X.; Lin, S.C.S.; Dong, C. Single-layer planar on-chip flow cytometer using microfluidic drifting based three-dimensional (3D) hydrodynamic focusing. Lab A Chip 2009, 9, 1583-1589. [CrossRef] [PubMed]

14. Lee, G.B.; Hung, C.I.; Ke, B.J. Hydrodynamic Focusing for a Micromachined Flow Cytometer. J. Fluids Eng. 2001, 123, 672. [CrossRef]

15. Lee, G.B.; Chang, C.C.; Huang, S.B. The hydrodynamic focusing effect inside rectangular microchannels. J. Micromechanics Microengineering 2006, 16, 1024. [CrossRef]

16. Oakey, J.; Applegate, R.W.; Arellano, E. Particle focusing in staged inertial microfluidic devices for flow cytometry. Anal. Chem. 2010, 82, 3862-3867. [CrossRef]

17. Zhao, J.; You, Z. Microfluidic hydrodynamic focusing for high-throughput applications. J. Micromechanics Microengineering 2015, 25, 125006. [CrossRef]

18. Stiles, T.; Fallon, R.; Vestad, T. Hydrodynamic focusing for vacuum-pumped microfluidics. Microfluid. Nanofluidics 2005, 1, 280-283. [CrossRef]

19. Cornish, R.J. Flow in a pipe of rectangular cross-section. Proc. R. Soc. Lond. Ser. A Contain. Pap. A Math. Phys. Character 1928, 120, 691-700.

20. Olson, R.; Sosik, H. A submersible imaging-in-flow instrument to analyze nano- and microplankton: Imaging FlowCytobot. Limnol. Oceanogr. Methods 2007, 5. [CrossRef] 
21. Carlo, D.; Edd, F.; Humphry, J.; Stone, H.A.; Toner, M. Particle Segregation and Dynamics in Confined Flows. Phys. Rev. Lett. 2009, 9, 102. [CrossRef] [PubMed]

22. Hayat, Z.; Abed, A. High-Throughput Optofluidic Acquisition of Microdroplets in Microfluidic Systems. Micromachines 2018, 9, 183. [CrossRef] [PubMed]

(C) 2020 by the authors. Licensee MDPI, Basel, Switzerland. This article is an open access article distributed under the terms and conditions of the Creative Commons Attribution (CC BY) license (http://creativecommons.org/licenses/by/4.0/). 